\title{
COPPER-CATALYZED SYNTHESIS OF 1,3-ENYNES
}

Craig G. Bates, Pranorm Saejueng, and D. Venkataraman*

Department of Chemistry, University of Massachusetts-Amherst, 710 N. Pleasant Street, Amherst, MA 01003.

\section{SUPPORTING INFORMATION}

General. All of the reactions reported herein were conducted under an inert atmosphere of argon in oven-dried glassware. All reagents and solvents were obtained from Acros, Alfa Aesar or from Aldrich and were used without further purification. Potassium Carbonate (Alfa Aesar, 99\%) was stored in an argon filled glove box. All vinyl iodides used in this paper have been synthesized using procedures previously reported in the literature. ${ }^{1-4}$ Purification was performed by flash chromatography using ICN Flash Silica Gel, 230-400 mesh. The yields given refer to isolated yields of the characterized compounds, deemed pure by elemental analyses, ${ }^{1} \mathrm{H}$ NMR and ${ }^{13} \mathrm{C}$ NMR. In certain cases GC yields were reported. All GC yields were calculated using dodecane as an internal standard; the correction factors used to calculate the product yields were determined using an analytically pure sample. NMR spectra were recorded on a Bruker AVANCE $400 \mathrm{MHz}$ spectrometer. Chemical shifts were reported in parts per million $(\delta)$. The peak patterns are indicated as follows: s, singlet; $d$, doublet; $t$, triplet; dd, doublet of doublets; dt, doublet of triplets; m, multiplet; and q, quartet. The coupling constants, J, are reported in Hertz $(\mathrm{Hz})$. TMS was used as the internal reference. Elemental analyses were performed at the Microanalysis Laboratory, University of Massachusetts - Amherst by Dr. Greg Dabkowski. The reported melting points were uncorrected. X-ray data were collected using a Nonius kappa-CCD diffractometer with MoKa $(\lambda=0.71073 \AA)$ as the incident radiation. Diffraction data were collected at ambient temperature. The raw data were integrated, refined, scaled and corrected for Lorentz polarization and absorption effects, if necessary, using the programs DENZO and SCALEPAK, supplied by Nonius. Structures solutions and refinements were done (on $\mathrm{F}_{\mathrm{o}}{ }^{2}$ ) using SIR92 and SHELXL 97 within the Nonius' MAXUS module. All structures were checked for any missing symmetry using MISSYM of PLATON. The Gas Chromatograph was a Hewlett Packard 6850 GC series with a 30-meter HP-1 100\% dimethylpolysiloxane capillary column 


\section{SYNTHESIS OF COPPER(I) COMPLEXES}

The following copper(I) complexes were synthesized using procedures outlined in literature: Nitratobis(triphenylphosphine)copper(I), Tris(triphenylphosphine)copper(I) bromide ${ }^{5} \quad\left[\mathrm{Cu}\left(\mathrm{CH}_{3} \mathrm{CN}\right)_{4}\right] \mathrm{PF}_{6},{ }^{6} \quad\left[\mathrm{Cu}(\right.$ phen $\left.)\left(\mathrm{PPh}_{3}\right) \mathrm{Br}\right],{ }^{5} \quad\left[\mathrm{Cu}(\right.$ phen $\left.)\left(\mathrm{PPh}_{3}\right)_{2}\right] \mathrm{NO}_{3},{ }^{5}$ [Cu(neocup)(PPh $)$ Br]. ${ }^{5}$

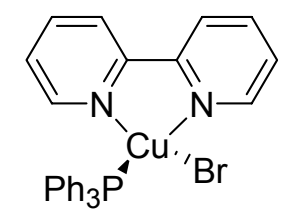

[Cu(bipy)(PPh $\left.\mathbf{P}_{3}\right) \mathrm{Br}$ ]: In a round bottom flask equipped with a Teflon-coated magnetic stir bar and reflux condenser, tris(triphenylphosphine)copper(I) bromide (7.663 g, 8.23 $\mathrm{mmol}$ ) was added to chloroform $(50 \mathrm{~mL})$. After complete dissolution, 2,2'-bipyridine (1.93 $\mathrm{g}, 12.37 \mathrm{mmol}$ ) was then added. The colorless solution immediately turned orange. The contents of the flask were allowed to reflux for 12 hours at $75^{\circ} \mathrm{C}$. Afterwards the solvent was removed in vacuo to afford an orange solid. Recrystallization was achieved by layering $80 \mathrm{~mL}$ of diethyl ether onto a solution of the solid dissolved in $30 \mathrm{~mL}$ of dichloromethane $\left(3.594 \mathrm{~g}, 78 \%\right.$ yield). m.p. $-198-200{ }^{\circ} \mathrm{C}$. The cell constants, contents and the space group are identical to that of the already reported structure of $\mathrm{Cu}\left(\right.$ bipy)$\left(\mathrm{PPh}_{3}\right) \mathrm{Br}$ (Cambridge Structural Database Refcode-COYNOT).

\section{SYNTHESIS OF 1,3-ENYNES:}

General Procedure: In an argon-filled glove box, a Pyrex glass tube $(2.5 \mathrm{~cm}$ in diameter) equipped with a Teflon-coated stir bar, was charged with potassium carbonate (Alfa Aesar, $0.553 \mathrm{~g}, 4.0 \mathrm{mmol})$ and [Cu(bipy) $\left(\mathrm{PPh}_{3}\right) \mathrm{Br}$ ] $(10 \mathrm{~mol} \%$ with respect to the acetylene). The tube was then sealed with a rubber septum, taken out of the glove box and toluene $(4.0 \mathrm{~mL}$ ) and $2.00 \mathrm{mmol}$ of the appropriate acetylene and $2.20 \mathrm{mmol}$ of the appropriate vinyl iodide were injected into the tube through the septum. The contents 
were then stirred at $110{ }^{\circ} \mathrm{C}$ for 8 hours unless specified otherwise. The reaction mixture was then cooled to room temperature and filtered through a pad of celite to remove any insoluble residues. The filtrate was concentrated in vacuo; the residue was purified by flash column chromatography on silica gel to obtain the analytically pure product.

Modified Procedure: In an argon-filled glove box, a Pyrex glass tube $(2.5 \mathrm{~cm}$ in diameter) equipped with a Teflon-coated stir bar, was charged with cesium carbonate (Aldrich, $1.303 \mathrm{~g}, 4.0 \mathrm{mmol})$ and $\left[\mathrm{Cu}(\right.$ phen $\left.)\left(\mathrm{PPh}_{3}\right)_{2} \mathrm{NO}_{3}\right](10 \mathrm{~mol} \%$ with respect to the acetylene). The tube was then sealed with a rubber septum, taken out of the glove box and toluene $(4.0 \mathrm{~mL})$ and $2.00 \mathrm{mmol}$ of the appropriate acetylene and $2.20 \mathrm{mmol}$ of the appropriate vinyl iodide were injected into the tube through the septum. The contents were then stirred at $110^{\circ} \mathrm{C}$ for 8 hours unless specified otherwise. The reaction mixture was then cooled to room temperature and filtered through a pad of celite to remove any insoluble residues. The filtrate was concentrated in vacuo; the residue was purified by flash column chromatography on silica gel to obtain the analytically pure product.

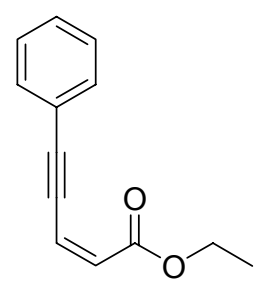

Ethyl (Z)-5-phenyl-2-buten-4-ynoate (Table 2, entry 1): The general procedure was used to convert phenylacetylene and (Z)-ethyl-3-iodoacrylate to the title product. Purification by flash chromatography (15\% ethyl acetate in hexanes as the eluent) gave the analytically pure product as a light yellow oil (396 mg, 99\% yield). ${ }^{1} \mathrm{H}$ NMR $(400 \mathrm{MHz}$, $\left.\mathrm{CDCl}_{3}\right) \delta 7.54-7.52(\mathrm{~m}, 2 \mathrm{H}), 7.34(\mathrm{~m}, 3 \mathrm{H}), 6.36(\mathrm{~d}, \mathrm{~J}=11.43,1 \mathrm{H}), 6.12(\mathrm{~d}, \mathrm{~J}=11.42,1 \mathrm{H})$, 4.26 (q, 2H), 1.33 (t, 3H). ${ }^{13} \mathrm{C}$ NMR (100 MHz, $\left.\mathrm{CDCl}_{3}\right) \delta 164.72,131.96,129.10,128.30$, 128.17, 122.75, 122.57, 101.10, 86.30, 60.36, 14.23. Anal. Calc'd. for $\mathrm{C}_{13} \mathrm{H}_{12} \mathrm{O}_{2}$ : C, 77.98; H, 6.04; Found C, 77.78; H, 6.06. 


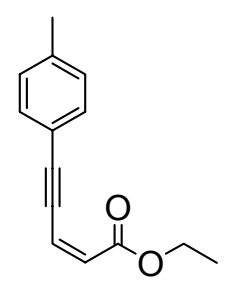

5-p-Tolyl-pent-2-en-4-ynoic acid ethyl ester (Table 2, entry 2): The general procedure was used to convert 4-ethynyltoluene and (Z)-ethyl-3-iodoacrylate to the title product. Purification by flash chromatography $(10 \%$ ethyl acetate in hexane as the eluent) gave the analytically pure product as a light yellow oil (350 mg, 85\% yield). ${ }^{1} \mathrm{H}$ NMR (400 MHz, $\left.\mathrm{CDCl}_{3}\right) \delta$ 7.43-7.41 (d, J= 8.1, 2H), 7.16-7.14 (d, J= 7.9, 2H), 6.37-6.34 $(\mathrm{d}, \mathrm{J}=11.4,1 \mathrm{H}), 6.12-6.09$ (d, J=11.4, 1H), 4.29-4.23 (q, J= 7.1, 2H), $2.36(\mathrm{~s}, 3 \mathrm{H}), 1.35-$ 1.31 (t, J=7.1,3H). ${ }^{13} \mathrm{C}$ NMR $\left(100 \mathrm{MHz} \mathrm{CDCl}_{3}\right) \delta 164.92,139.62,132.12,129.28$, 127.83, 123.14, 119.76, 101.77, 86.19, 60.44, 21.66, 14.42. Anal. Calcd. for $\mathrm{C}_{14} \mathrm{H}_{14} \mathrm{O}_{2}$ : C, 78.48; H, 6.59; Found C, 78.22; H, 6.78.

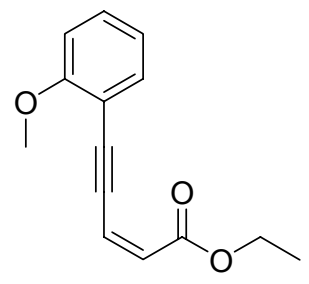

5-(2-Methoxy-phenyl)-pent-2-en-4-ynoic acid ethyl ester (Table 2, entry 3): The general procedure was used to convert 2-ethynylanisole and (Z)-ethyl-3-iodoacrylate to the title product. Purification by flash chromatography $(20 \%$ ethyl acetate in hexane as the eluent) gave the analytically pure product as a light yellow oil (480 mg, 98\% yield). ${ }^{1} \mathrm{H}$ NMR (400 MHz, $\left.\mathrm{CDCl}_{3}\right) \delta$ 7.52-7.50 (dd, J= 7.5, 1.7, 1H), 7.33-7.29 (m, 1H), 6.93-6.89 $(\mathrm{dt}, \mathrm{J}=7.6,0.9,1 \mathrm{H}), 6.88-6.86(\mathrm{~d}, \mathrm{~J}=8.3,1 \mathrm{H}), 6.42-6.39(\mathrm{~d}, \mathrm{~J}=11.4,1 \mathrm{H}), 6.11-6.08(\mathrm{~d}$, $\mathrm{J}=11.4,1 \mathrm{H}), 4.28-4.22(\mathrm{q}, \mathrm{J}=7.1,2 \mathrm{H}), 3.88(\mathrm{~s}, 3 \mathrm{H}), 1.33-1.29(\mathrm{t}, \mathrm{J}=7.1,3 \mathrm{H}) .{ }^{13} \mathrm{C} N M R$ $\left(100 \mathrm{MHz}, \mathrm{CDCl}_{3}\right) \delta 164.86,160.32,134.25,130.84,127.67,123.10,120.54,111.96$, 110.73, 97.93, 90.45, 60.32, 55.81, 14.28. Anal. Calcd. for $\mathrm{C}_{14} \mathrm{H}_{14} \mathrm{O}_{3}: \mathrm{C}, 73.03 ; \mathrm{H}, 6.13$; Found C, 73.08; H, 6.19. 


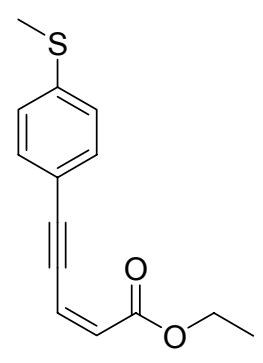

5-(4-Methylsulfanyl-phenyl)-pent-2-en-4-ynoic acid ethyl ester (Table 2, entry 4): The general procedure was used to convert 4-ethynylthioanisole and ( $Z$ )-ethyl-3iodoacrylate to the title product. Purification by flash chromatography $(20 \%$ ethyl acetate in hexane as the eluent) gave the analytically pure product as a light yellow oil $(450 \mathrm{mg}$, 91\% yield). ${ }^{1} \mathrm{H}$ NMR $\left(400 \mathrm{MHz}, \mathrm{CDCl}_{3}\right) \delta$ 7.44-7.42 (d, J= 8.5, 2H), 7.18-7.16 (d, J= 8.5, $2 \mathrm{H}), 6.35-6.32(\mathrm{~d}, \mathrm{~J}=11.4,1 \mathrm{H}), 6.11-6.09(\mathrm{~d}, \mathrm{~J}=11.4,1 \mathrm{H}), 4.28-4.22(\mathrm{q}, \mathrm{J}=7.1,2 \mathrm{H})$, $2.46(\mathrm{~s}, 3 \mathrm{H}), 1.34-1.30(\mathrm{t}, \mathrm{J}=7.1,3 \mathrm{H}) .{ }^{13} \mathrm{C} \mathrm{NMR}\left(100 \mathrm{MHz}, \mathrm{CDCl}_{3}\right) \delta 164.81,140.87$, 132.33, 127.85, 125.68, 122.81, 118.84, 101.21, 86.77, 60.37, 15.16, 14.32. Anal. Calcd. for $\mathrm{C}_{14} \mathrm{H}_{14} \mathrm{O}_{2} \mathrm{~S}$ : C, 68.26; H, 5.73; S, 13.02; Found C, 68.50; H, 5.88; S, 13.18 .

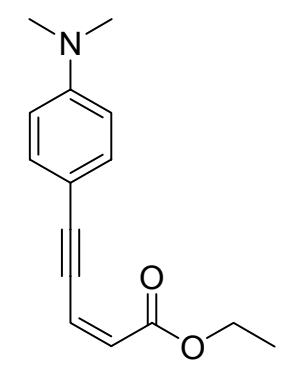

5-(4-Dimethylamino-phenyl)-pent-2-en-4-ynoic acid ethyl ester (Table 2, entry 5): The general procedure was used to convert 4-ethynyl- $N, N$-dimethylaniline and (Z)-ethyl3 -iodoacrylate to the title product. Purification by flash chromatography $(10 \%$ ethyl acetate in hexane as the eluent) gave the analytically pure product as a yellow solid (420 $\mathrm{mg}, 88 \%$ yield). ${ }^{1} \mathrm{H}$ NMR $\left(400 \mathrm{MHz}, \mathrm{CDCl}_{3}\right) \delta$ 7.42-7.39 (d, J=8.9, 2H), 6.62-6.60 (d, J= 9.0, 2H), 6.37-6.34 (d, J=11.4, 1H), 6.01-5.98 (d, $J=11.4,1 \mathrm{H}), 4.28-4.22(\mathrm{q}, \mathrm{J}=7.1,2 \mathrm{H})$, $2.97(\mathrm{~s}, 6 \mathrm{H}), 1.35-1.31(\mathrm{t}, \mathrm{J}=7.1,3 \mathrm{H}) .{ }^{13} \mathrm{C} \mathrm{NMR}\left(100 \mathrm{MHz}, \mathrm{CDCl}_{3}\right) \delta 165.15,150.62$, 133.53, 125.21, 123.63, 111.48, 108.97, 104.06, 85.92, 60.08, 39.94, 14.28. Anal. Calcd. for $\mathrm{C}_{15} \mathrm{H}_{17} \mathrm{NO}_{2}$ : C, 74.05; H, 7.04; N, 5.76; Found C, 74.29; H, 7.20; N, 5.71. m.p.: $58.0^{\circ}-59.5^{\circ} \mathrm{C}$. 


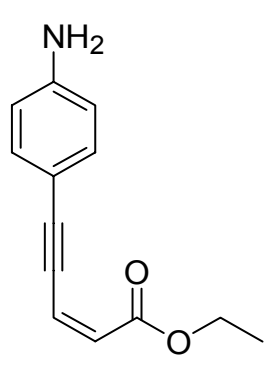

5-(4-Amino-phenyl)-pent-2-en-4-ynoic acid ethyl ester (Table 2, entry 6): The general procedure was used to convert 4-ethynylaniline and (Z)-ethyl-3-iodoacrylate to the title product. Purification by flash chromatography $(30 \%$ ethyl acetate in hexane as the eluent) gave the analytically pure product as a light yellow oil (350 mg, 90\% yield). ${ }^{1} \mathrm{H}$ NMR (400 MHz, $\left.\mathrm{CDCl}_{3}\right) \delta$ 7.34-7.32 (d, J= 8.4, 2H), 6.60-6.58 (d, J=8.4, 2H), 6.36-6.34 $(\mathrm{d}, \mathrm{J}=11.4,1 \mathrm{H}), 6.05-6.02(\mathrm{~d}, \mathrm{~J}=11.4,1 \mathrm{H}), 4.28-4.23$ (q, J= 7.1, 2H), $3.96(\mathrm{~s}, 2 \mathrm{H}), 1.35-$ $1.31(\mathrm{t}, \mathrm{J}=7.1,3 \mathrm{H}) .{ }^{13} \mathrm{C} \mathrm{NMR}\left(100 \mathrm{MHz}, \mathrm{CDCl}_{3}\right) \delta 165.21,148.01,133.78,125.92$, 123.66, 114.54, 111.33, 103.46, 85.59, 60.28, 14.32. Anal. Calcd. for $\mathrm{C}_{13} \mathrm{H}_{13} \mathrm{NO}_{2}$ : C, 72.54; H, 6.09; N, 6.51; Found C, 72.63; H, 6.26; N, 6.32.

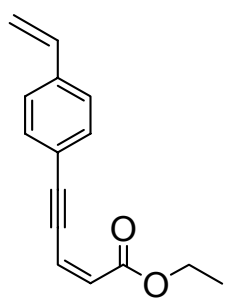

5-(4-Vinyl-phenyl)-pent-2-en-4-ynoic acid ethyl ester (Table 2, entry 7): The general procedure was used to convert 4-ethynylstyrene and $(Z)$-ethyl-3-iodoacrylate to the title product. Purification by flash chromatography $(10 \%$ ethyl acetate in hexane as the eluent) gave the analytically pure product as a light yellow oil (420 mg, 95\% yield). ${ }^{1} \mathrm{H}$ NMR (400 MHz, $\mathrm{CDCl}_{3}$ ) $\delta$ 7.51-7.49 (d, J= 8.3, 2H), 7.39-7.37 (d, J= 8.3, 2H), 6.73-6.66 $(\mathrm{dd}, \mathrm{J}=17.5,10.8,1 \mathrm{H}), 6.38-6.35(\mathrm{~d}, \mathrm{~J}=11.4,1 \mathrm{H}), 6.14-6.12(\mathrm{~d}, \mathrm{~J}=11.4,1 \mathrm{H}), 5.81-5.77$ $(\mathrm{d}, \mathrm{J}=17.6,1 \mathrm{H}), 5.33-5.30(\mathrm{~d}, \mathrm{~J}=10.9,1 \mathrm{H}), 4.29-4.24(\mathrm{q}, \mathrm{J}=7.1,2 \mathrm{H}), 1.35-1.32(\mathrm{t}$, $\mathrm{J}=7.1,3 \mathrm{H}) .{ }^{13} \mathrm{C}$ NMR $\left(100 \mathrm{MHz}, \mathrm{CDCl}_{3}\right) \delta 164.92,139.62,132.12,129.28,127.83$, 123.14, 119.76, 101.77, 86.19, 60.44, 21.66, 14.42. Anal. Calcd. for $\mathrm{C}_{14} \mathrm{H}_{14} \mathrm{O}_{2}$ : C, 79.62; H, 6.24; Found C, 79.37; H, 6.48. 


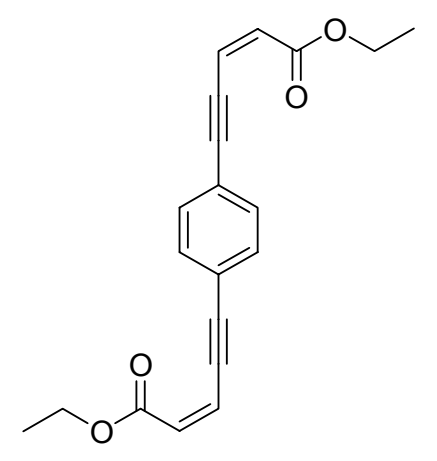

\section{5-[4-(4-Ethoxycarbonyl-but-3-en-1-ynyl)-phenyl]-pent-2-en-4-ynoic acid ethyl ester}

(Table 2, entry 8): The general procedure was used to convert 1,4-diethynylbenzene and (Z)-ethyl-3-iodoacrylate to the title product in 12 hours. Purification by flash chromatography (10\% ethyl acetate in hexane as the eluent) gave the analytically pure product as a light yellow solid (560 mg, 87\% yield). ${ }^{1} \mathrm{H}$ NMR $\left(400 \mathrm{MHz}, \mathrm{CDCl}_{3}\right) \delta 7.50$ (s, $4 \mathrm{H}), 6.37-6.34(\mathrm{~d}, \mathrm{~J}=11.4,2 \mathrm{H}), 6.17-6.14(\mathrm{~d}, \mathrm{~J}=11.4,2 \mathrm{H}), 4.28-4.23(\mathrm{q}, \mathrm{J}=7.1,4 \mathrm{H})$, 1.34-1.30 (t, $\mathrm{J}=7.1,6 \mathrm{H}) .{ }^{13} \mathrm{C}$ NMR $\left(100 \mathrm{MHz}, \mathrm{CDCl}_{3}\right) \delta 164.57,131.88,128.72,123.31$, 122.34, 100.27, 88.36, 60.39, 14.21. Anal. Calcd. for $\mathrm{C}_{20} \mathrm{H}_{18} \mathrm{O}_{4}$ : C, 74.52; $\mathrm{H}, 5.63$; Found C, 74.33; H, 5.71. m.p.: $73.0^{\circ}-74.0^{\circ} \mathrm{C}$.

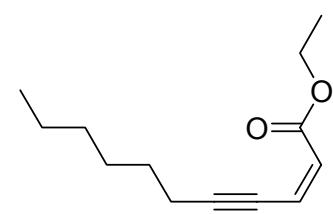

(Z)-ethyl undec-2-en-4-ynoate (Table 2. entry 9): The general procedure was used to convert $n$-octyne and $(Z)$-ethyl-3-iodoacrylate to the title product. Purification by flash chromatography ( $5 \%$ ethyl acetate in hexane as the eluent) gave the analytically pure product as a light yellow oil (401 mg, 96\% yield). ${ }^{1} \mathrm{H}$ NMR (400 MHz, $\left.\mathrm{CDCl}_{3}\right) \delta 6.13$ (dt, $J=10.82,1 \mathrm{H}), 6.02(\mathrm{~d}, \mathrm{~J}=10.96,1 \mathrm{H}), 4.21(\mathrm{q}, 2 \mathrm{H}), 2.44(\mathrm{~m}, 2 \mathrm{H}), 1.58(\mathrm{p}, 2 \mathrm{H}), 1.42(\mathrm{~m}$, $2 \mathrm{H}), 1.30-1.28(\mathrm{~m}, 7 \mathrm{H}), 0.89(\mathrm{t}, 3 \mathrm{H}) .{ }^{13} \mathrm{C} \operatorname{NMR}\left(100 \mathrm{MHz}, \mathrm{CDCl}_{3}\right) \delta 164.87,127.34$, 123.89, 104.17, 77.66, 60.18, 31.30, 28.60, 28.36, 22.49, 20.07, 14.21, 13.99. Anal. Calcd. for $\mathrm{C}_{13} \mathrm{H}_{20} \mathrm{O}_{2}$ : C, 74.96; H, 9.68; Found C, 74.96; H, 9.56. 


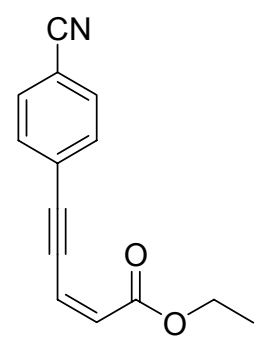

5-(4-Cyano-phenyl)-pent-2-en-4-ynoic acid ethyl ester (Table 2, entry 10): The general procedure was used to convert 4-ethynylbezonitrile and (Z)-ethyl-3-iodoacrylate to the title product. Purification by flash chromatography $(20 \%$ ethyl acetate in hexane as the eluent) gave the analytically pure product as a light yellow solid ( $380 \mathrm{mg}, 85 \%$ yield). ${ }^{1} \mathrm{H}$ NMR $\left(400 \mathrm{MHz}, \mathrm{CDCl}_{3}\right) \delta$ 7.64-7.59 (m, J= 8.5, 4H), 6.37-6.34 (d, J=11.3, $\left.1 \mathrm{H}\right), 6.24-$ $6.21(\mathrm{~d}, \mathrm{~J}=11.3,1 \mathrm{H}), 4.28-4.23(\mathrm{q}, \mathrm{J}=7.1,2 \mathrm{H}), 1.34-1.30(\mathrm{t}, \mathrm{J}=7.1,3 \mathrm{H}) .{ }^{13} \mathrm{C}$ NMR $(100$ $\left.\mathrm{MHz}, \mathrm{CDCl}_{3}\right) \delta 164.40,132.39,132.03,130.08,127.49,121.74,118.25,112.37,98.34$, 89.94, 60.57, 14.23. Anal. Calcd. for $\mathrm{C}_{14} \mathrm{H}_{11} \mathrm{NO}_{2}$ : C, 74.65; H, 4.92, N, 6.22; Found C, 74.45; H, 4.84, N, 6.06. m.p.: $69.5^{\circ}-71.5^{\circ} \mathrm{C}$.

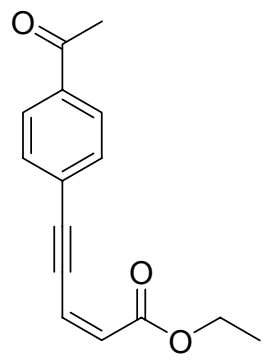

5-(4-Acetyl-phenyl)-pent-2-en-4-ynoic acid ethyl ester (Table 2, entry 11): The general procedure was used to convert 4-ethynylacetophenone and (Z)-ethyl-3iodoacrylate to the title product. Purification by flash chromatography ( $440 \mathrm{mg}, 20 \%$ ethyl acetate in hexane as the eluent) gave the analytically pure product as a light yellow oil (92\% yield). ${ }^{1} \mathrm{H}$ NMR (400 MHz, $\left.\mathrm{CDCl}_{3}\right) \delta$ 7.93-7.90 (d, J= 8.5, 2H), 7.60-7.58 (d, J= 8.5, $2 \mathrm{H}), 6.38-6.35(\mathrm{~d}, \mathrm{~J}=11.4,1 \mathrm{H}), 6.20-6.18(\mathrm{~d}, \mathrm{~J}=11.4,1 \mathrm{H}), 4.28-4.23(\mathrm{q}, \mathrm{J}=7.1,2 \mathrm{H})$, $2.58(\mathrm{~s}, 3 \mathrm{H}), 1.34-1.30(\mathrm{t}, \mathrm{J}=7.1,3 \mathrm{H}) .{ }^{13} \mathrm{C} \mathrm{NMR}\left(100 \mathrm{MHz}, \mathrm{CDCl}_{3}\right) \delta 197.09,164.54$, 136.87, 132.09, 129.43, 128.24, 127.39, 122.22. 99.65, 89.11, 60.53, 26.59, 14.29. Anal. Calcd. for $\mathrm{C}_{15} \mathrm{H}_{14} \mathrm{O}_{3}: \mathrm{C}, 74.36 ; \mathrm{H}, 5.82$; Found $\mathrm{C}, 74.31 ; \mathrm{H}, 5.97$. 


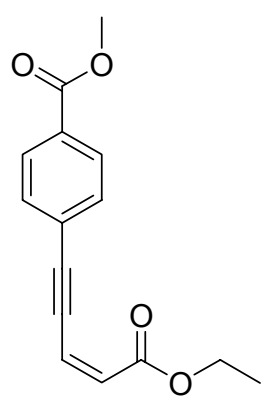

\section{4-(4-Ethoxycarbonyl-but-3-en-1-ynyl)-benzoic acid methyl ester (Table 2, entry 12):}

The general procedure was used to convert 4-ethynyl-benzoic acid methyl ester and $(Z)$ ethyl-3-iodoacrylate to the title product. Purification by flash chromatography ( $30 \%$ ethyl acetate in hexane as the eluent) gave the analytically pure product as a light yellow solid (450 mg, 88\% yield). ${ }^{1} \mathrm{H}$ NMR (400 MHz, $\left.\mathrm{CDCl}_{3}\right) \delta 8.01-7.99$ (d, J= 8.5, 2H), 7.59-7.57 $(\mathrm{d}, \mathrm{J}=8.5,2 \mathrm{H}), 6.37-6.34(\mathrm{~d}, \mathrm{~J}=11.4,1 \mathrm{H}), 6.20-6.17(\mathrm{~d}, \mathrm{~J}=11.4,1 \mathrm{H}), 4.29-4.23$ (q, $\mathrm{J}=$ 7.1, 2H), $3.91(\mathrm{~s}, 3 \mathrm{H}), 1.34-1.30(\mathrm{t}, \mathrm{J}=7.1,3 \mathrm{H}) .{ }^{13} \mathrm{C} \mathrm{NMR}\left(100 \mathrm{MHz}, \mathrm{CDCl}_{3}\right) \delta 166.36$, $164.60,131.91,130.31,129.52,129.37,127.29,122.25,99.73,88.79,60.55,52.24$, 14.30. Anal. Calcd. for $\mathrm{C}_{15} \mathrm{H}_{14} \mathrm{O}_{4}$ : C, 69.76; H, 5.46; Found C, 69.90; H, 5.55. m.p.: 49.0 $51.0^{\circ} \mathrm{C}$.

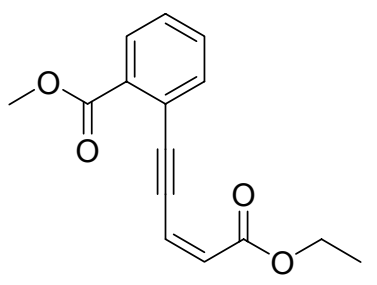

2-(4-Ethoxycarbonyl-but-3-en-1-ynyl)-benzoic acid methyl ester (Table 2, entry 13): The general procedure was used to convert 2-ethynyl-benzoic acid methyl ester and $(Z)$ ethyl-3-iodoacrylate to the title product. Purification by flash chromatography $(20 \%$ ethyl acetate in hexane as the eluent) gave the analytically pure product as a light yellow oil (410 mg, 77\% yield). ${ }^{1} \mathrm{H}$ NMR (400 MHz, $\left.\mathrm{CDCl}_{3}\right) \delta 7.96-7.95$ (d, J= 7.8, 1H), 7.70-7.68 $(\mathrm{d}, \mathrm{J}=8.2,1 \mathrm{H}), 7.50-7.46(\mathrm{t}, J=7.5,1 \mathrm{H}), 7.37-7.41(\mathrm{t}, \mathrm{J}=7.7,1 \mathrm{H}), 6.46-6.43(\mathrm{~d}, \mathrm{~J}=11.4$, $1 \mathrm{H}), 6.18-6.15(\mathrm{~d}, \mathrm{~J}=11.4,1 \mathrm{H}), 4.27-4.21(\mathrm{q}, \mathrm{J}=7.1,2 \mathrm{H}), 3.92(\mathrm{~s}, 3 \mathrm{H}), 1.31-1.27(\mathrm{t}, \mathrm{J}=$ $7.1,3 \mathrm{H}) .{ }^{13} \mathrm{C}$ NMR $\left(100 \mathrm{MHz}, \mathrm{CDCl}_{3}\right) \delta 166.31,164.65,134.69,131.79,131.74,130.42$, 128.73, 128.70, 123.23, 122.91, 99.64, 90.93, 60.40, 52.23, 14.27. Anal. Calcd. for $\mathrm{C}_{15} \mathrm{H}_{14} \mathrm{O}_{4}: \mathrm{C}, 69.76 ; \mathrm{H}, 5.46$; Found $\mathrm{C}, 69.93 ; \mathrm{H}, 5.59$. 


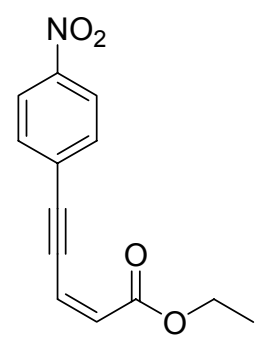

5-(4-Nitro-phenyl)-pent-2-en-4-ynoic acid ethyl ester (Table 2, entry 14): The general procedure was used to convert 4-ethynylnitrobenzene and (Z)-ethyl-3iodoacrylate to the title product except using $1.6 \mathrm{mmol}$ of acetylene and $1.8 \mathrm{mmol}$ vinyl iodide. Purification by flash chromatography (20\% ethyl acetate in hexane as the eluent) gave the analytically pure product as a yellow solid (350 mg, 89\% yield). ${ }^{1} \mathrm{H}$ NMR (400 $\left.\mathrm{MHz}, \mathrm{CDCl}_{3}\right) \delta$ 8.22-8.19 (d, J= 8.9, 2H), 7.68-7.65 (d, J=8.9, 2H), 6.38-6.35 (d, J= 11.4, $1 \mathrm{H}), 6.26-6.23(\mathrm{~d}, \mathrm{~J}=11.4,1 \mathrm{H}), 4.29-4.24$ (q, $J=7.1,2 \mathrm{H}), 1.35-1.31(\mathrm{t}, J=7.1,3 \mathrm{H}) .{ }^{13} \mathrm{C}$ NMR $\left(100 \mathrm{MHz}, \mathrm{CDCl}_{3}\right) \delta 164.38,147.48,132.66,130.32,129.41,123.57,121.67$, 97.99, 90.64, 60.64, 14.23. Anal. Calcd. for $\mathrm{C}_{13} \mathrm{H}_{11} \mathrm{NO}_{4}: \mathrm{C}, 63.67 ; \mathrm{H}, 4.52 ; \mathrm{N}, 5.71$; Found C, 63.45; H, 4.44; N, 5.65. m.p.: $76.0^{\circ}-78.0^{\circ} \mathrm{C}$.

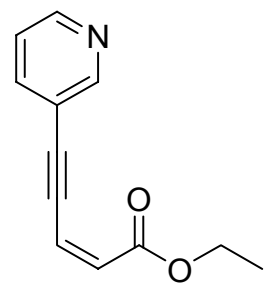

5-Pyridin-3-yl-pent-2-en-4-ynoic acid ethyl ester (Table 2, entry 15): The modified procedure was used to convert 3-ethynylpyridine and (Z)-ethyl-3-iodoacrylate to the title product in 12 hours. Purification by flash chromatography (30\% ethyl acetate in hexane as the eluent) gave the analytically pure product as a light yellow oil (200 $\mathrm{mg}, 51 \%$ yield). ${ }^{1} \mathrm{H}$ NMR $\left(400 \mathrm{MHz}, \mathrm{CDCl}_{3}\right) \delta 8.76(\mathrm{~s}, 1 \mathrm{H}), 8.57-8.55(\mathrm{dd}, \mathrm{J}=4.9,1.5,1 \mathrm{H}), 7.82-$ $7.79(\mathrm{td}, \mathrm{J}=7.8,1.8,1 \mathrm{H}), 7.29-7.26(\mathrm{~m}, 1 \mathrm{H}), 6.38-6.35(\mathrm{~d}, \mathrm{~J}=11.4,1 \mathrm{H}), 6.21-6.18(\mathrm{~d}, \mathrm{~J}=$ $11.4,1 \mathrm{H}$ ), 4.29-4.23 (q, J=7.1, 2H), 1.34-1.31 (t, J=7.1, 3H). ${ }^{13} \mathrm{C}$ NMR (100 MHz, $\left.\mathrm{CDCl}_{3}\right)$ $\delta 164.32,152.25,149.13,138.58,129.22,122.86,121.80,119.68,96.97,89.03,60.34$, 14.08. Anal. Calcd. for $\mathrm{C}_{12} \mathrm{H}_{11} \mathrm{NO}_{2}$ : C, 71.63; H, 5.51; N, 6.96; Found C, 71.77; H, 5.64; $\mathrm{N}, 6.73$. 


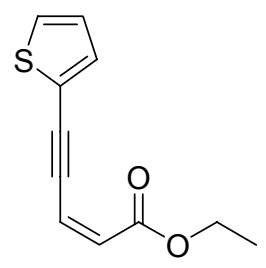

5-Thiophen-2-yl-pent-2-en-4-ynoic acid ethyl ester (Table 2, entry 16): The general procedure was used to convert 2-ethynylthiophene and (Z)-ethyl-3-iodoacrylate to the title product. Purification by flash chromatography $(10 \%$ ethyl acetate in hexane as the eluent) gave the analytically pure product as a light yellow oil (230 mg, $62 \%$ yield). ${ }^{1} \mathrm{H}$ $\operatorname{NMR}\left(400 \mathrm{MHz}, \mathrm{CDCl}_{3}\right) \delta$ 7.36-7.35 (dd, J= 5.1, 1.0, 1H), 7.33-7.32 (dd, J= 3.6, 1.0, $\left.1 \mathrm{H}\right)$, 7.02-7.00 (m, 1H), 6.36-6.33 (d, J= 11.4, 1H), 6.10-6.08 (d, J= 11.4, 1H), 4.29-4.23 (q, $J=7.1,2 \mathrm{H}), 1.36-1.32(\mathrm{t}, J=7.1,3 \mathrm{H}) .{ }^{13} \mathrm{C} \mathrm{NMR}\left(100 \mathrm{MHz}, \mathrm{CDCl}_{3}\right) \delta 164.79,133.49$, 129.19, 127.62, 127.36, 122.57, 122.29, 94.50, 90.82, 60.51, 14.31. Anal. Calcd. for $\mathrm{C}_{11} \mathrm{H}_{10} \mathrm{O}_{2} \mathrm{~S}$ : C, 64.05; H, 4.89; S, 15.55; Found C, 64.01; H, 4.95; S, 15.27.

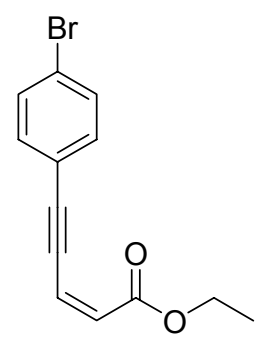

5-(4-Bromo-phenyl)-pent-2-en-4-ynoic acid ethyl ester (Table 2, entry 17): The general procedure was used to convert 1-ethynyl-4-bromobenzene and (Z)-ethyl-3iodoacrylate to the title product in 20 hours. Purification by flash chromatography (355 $\mathrm{mg}, 20 \%$ ethyl acetate in hexane as the eluent) gave the analytically pure product as a light yellow oil (65\% yield). ${ }^{1} \mathrm{H}$ NMR (400 MHz, $\left.\mathrm{CDCl}_{3}\right) \delta 7.48-7.46(\mathrm{~d}, \mathrm{~J}=8.5,2 \mathrm{H}), 7.39$ $7.36(\mathrm{~d}, \mathrm{~J}=8.5,2 \mathrm{H}), 6.33-6.30(\mathrm{~d}, \mathrm{~J}=11.4,1 \mathrm{H}), 6.16-6.13(\mathrm{~d}, \mathrm{~J}=11.4,1 \mathrm{H}), 4.27-4.22$ (q, $J=7.1,2 \mathrm{H}), 1.33-1.29(\mathrm{t}, J=7.1,3 \mathrm{H}) .{ }^{13} \mathrm{C} \mathrm{NMR}\left(100 \mathrm{MHz}, \mathrm{CDCl}_{3}\right) \delta 164.64,133.40$, 131.68, 128.69, 123.61, 122.49, 121.60, 99.81, 87.38, 60.46, 14.29. Anal. Calcd. for $\mathrm{C}_{13} \mathrm{H}_{11} \mathrm{BrO}_{2}$ : C, 55.94; H, 3.97; $\mathrm{Br}, 28.63$; Found C, 56.05; H, 4.07; Br, 27.6. 


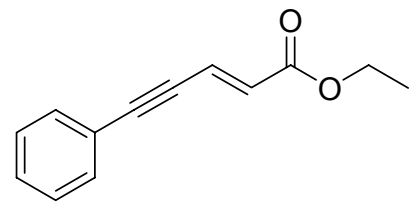

Ethyl (E)-5-phenyl-2-buten-4-ynoate (Table 3, entry 1): The general procedure was used to convert phenylacetylene and $(E)$-ethyl-3-iodoacrylate to the title product in 24 hours. Purification by flash chromatography (10\% ethyl acetate in hexanes as the eluent) gave the analytically pure product as a light yellow oil (325 mg, 81\% yield). ${ }^{1} \mathrm{H}$ NMR (400 $\mathrm{MHz}_{\mathrm{CDCl}}$ ) $\delta 7.47(\mathrm{~m}, 2 \mathrm{H}), 7.35(\mathrm{~m}, 3 \mathrm{H}), 6.98(\mathrm{~d}, \mathrm{~J}=15.84,1 \mathrm{H}), 6.30(\mathrm{~d}, \mathrm{~J}=15.84,2 \mathrm{H})$, 4.24 (q, 2H), 1.31 (t, 3H). ${ }^{13} \mathrm{C}$ NMR (100 MHz, $\left.\mathrm{CDCl}_{3}\right) \delta 165.84,131.91,130.01,129.24$, 128.41, 125.01, 122.162, 98.20, 86.32, 60.72, 14.19. Anal. Calc'd. for $\mathrm{C}_{13} \mathrm{H}_{12} \mathrm{O}_{2}$ : C, 77.98; H, 6.04; Found C, 78.06; H, 6.13.

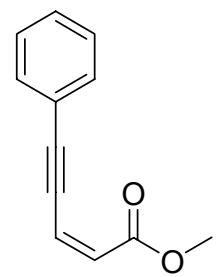

Methyl (Z)-5-phenyl-2-penten-4-ynoate (Table 3, entry 2): The general procedure was used to convert phenylacetylene and (Z)-methyl-3-iodoacrylate to the title product. Purification by flash chromatography (15\% ethyl acetate in hexanes as the eluent) gave the analytically pure product as a light yellow oil (336 mg, 90\% yield). ${ }^{1} \mathrm{H}$ NMR $(400 \mathrm{MHz}$, $\left.\mathrm{CDCl}_{3}\right) \delta 7.54(\mathrm{~m}, 2 \mathrm{H}), 7.35(\mathrm{~m}, 3 \mathrm{H}), 6.36(\mathrm{~d}, \mathrm{~J}=11.39,1 \mathrm{H}), 6.14(\mathrm{~d}, \mathrm{~J}=11.41,1 \mathrm{H}), 3.80$ (s, 3H). ${ }^{13} \mathrm{C}$ NMR $\left(100 \mathrm{MHz}, \mathrm{CDCl}_{3}\right) \delta 165.25,132.16,129.28,128.42,127.79,123.22$, 122.63, 101.45, 86.37, 51.55. Anal. Calc'd. for $\mathrm{C}_{12} \mathrm{H}_{10} \mathrm{O}_{2}: \mathrm{C}, 77.40 ; \mathrm{H}, 5.41$; Found $\mathrm{C}$, $77.41 ; \mathrm{H}, 5.35$.

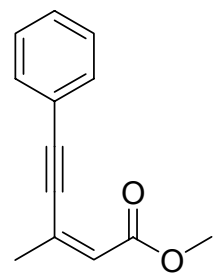


cis-3-methyl-5-phenyl-pent-2-en-4-ynoic acid methyl ester (Table 3, entry 3): The general procedure was used to convert phenylacetylene and (Z)- $\beta$-lodo- $\beta$-methyl methyl acrylate to the title product. Purification by flash chromatography $(15 \%$ ethyl acetate in hexanes as the eluent) gave the analytically pure product as a light yellow oil (388 $\mathrm{mg}$, 97\% yield). ${ }^{1} \mathrm{H}$ NMR (400 MHz, $\left.\mathrm{CDCl}_{3}\right) \delta 7.55(\mathrm{~m}, 2 \mathrm{H}), 7.33(\mathrm{~m}, 3 \mathrm{H}), 6.03$ (q, 1H), 3.76 $(\mathrm{s}, 3 \mathrm{H}), 2.13$ (d, J= 1.45, 3H). ${ }^{13} \mathrm{C}$ NMR $\left(100 \mathrm{MHz}, \mathrm{CDCl}_{3}\right) \delta 165.42,134.99,132.03$, 129.02, 128.29, 123.85, 122.65, 100.31, 88.25, 51.23, 25.11. Anal. Calc'd. for $\mathrm{C}_{13} \mathrm{H}_{12} \mathrm{O}_{2}$ : C, 77.98; H, 6.04; Found C, 77.83; H, 6.04.

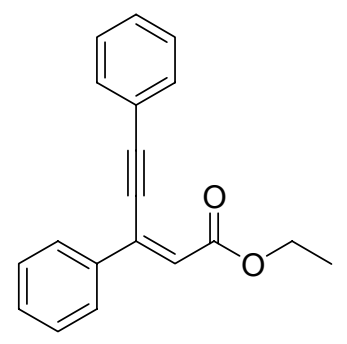

(Z)-ethyl 3,5-diphenylpent-2-en-4-ynoate (Table 3, entry 4): The general procedure was used to convert phenylacetylene and (Z)-ethyl 3-iodo-3-phenylacrylate to the title product. Purification by flash chromatography (5\% ethyl acetate in hexanes as the eluent) gave the analytically pure product as a light yellow oil (530 mg, 96\% yield). ${ }^{1} \mathrm{H}$ NMR (400 MHz, $\left.\mathrm{CDCl}_{3}\right) \delta 7.78(\mathrm{~m}, 2 \mathrm{H}), 7.63(\mathrm{~m}, 2 \mathrm{H}), 7.38(\mathrm{~m}, 6 \mathrm{H}), 6.59(\mathrm{~s}, 1 \mathrm{H}), 2.06$ (q, $2 \mathrm{H}), 1.35$ (t, 3H). ${ }^{13} \mathrm{C}$ NMR $\left(100 \mathrm{MHz}, \mathrm{CDCl}_{3}\right) \delta 165.12,136.92,136.08,131.85,129.66$, 128.99, 128.42, 128.18, 126.96, 122.54, 122.48, 101.88, 86.68, 60.16, 14.17. Anal. Calc'd. for $\mathrm{C}_{19} \mathrm{H}_{16} \mathrm{O}_{2}$ : C, 82.58; $\mathrm{H}, 5.84$; Found $\mathrm{C}$,

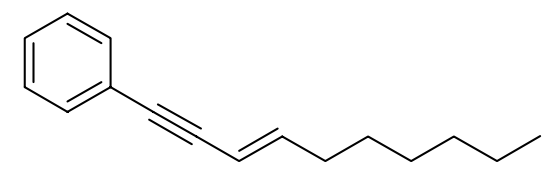

(E)-1-Phenyldec-3-en-1-yne (Table 3, entry 5): The general procedure was used to convert phenylacetylene and $(E)$-1-iodooctene to the title product in 24 hours. Purification by flash chromatography (light petroleum ether as eluent) gave the analytically pure product as a clear oil $\left(423 \mathrm{mg}, 99 \%\right.$ yield). ${ }^{1} \mathrm{H} \mathrm{NMR}\left(400 \mathrm{MHz}, \mathrm{CDCl}_{3}\right)$ $\delta 7.40(\mathrm{~m}, 2 \mathrm{H}), 7.28(\mathrm{~m}, 3 \mathrm{H}), 6.24(\mathrm{~m}, 1 \mathrm{H}), 5.68(\mathrm{~d}, \mathrm{~J}=15.84,1 \mathrm{H}), 2.15(\mathrm{q}, 2 \mathrm{H}), 1.41-1.28$ $(\mathrm{m}, 8 \mathrm{H}), 0.89(\mathrm{t}, 3 \mathrm{H}) .{ }^{13} \mathrm{C}$ NMR $\left(100 \mathrm{MHz}, \mathrm{CDCl}_{3}\right) \delta 145.08,131.24,128.07,127.67$, 
123.49, 109.32, 88.23, 87.68, 33.08, 31.52, 28.66, 28.59, 22.45, 13.94. Anal. Calc'd. for $\mathrm{C}_{16} \mathrm{H}_{20}: \mathrm{C}, 90.51 ; \mathrm{H}, 9.49$; Found $\mathrm{C}, 90.65 ; \mathrm{H}, 9.58$.

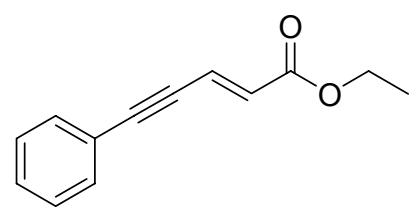

Ethyl (E)-5-phenyl-2-buten-4-ynoate (Table 4 entry 1): The modified procedure was used to convert phenylacetylene and $(E)$-ethyl-3-iodoacrylate to the title product. GC yield was found to be $74 \%$ and $99 \%$ after 8 and 24 hours respectively.

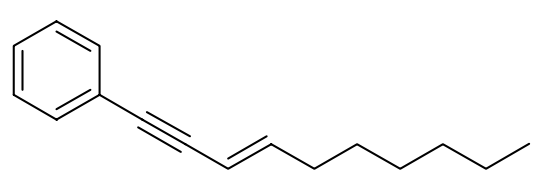

(E)-1-Phenyldec-3-en-1-yne (Table 4, entry 2): The modified procedure was used to convert phenylacetylene and $(E)-1$-iodooctene to the title product. Purification by flash chromatography (light petroleum ether as eluent) afforded a clear oil (418 mg, $98 \%$ yield). The proton spectra obtained matches that of the analytically pure compound previously isolated (see Table 3, entry 5). ${ }^{1} \mathrm{H} \mathrm{NMR}\left(400 \mathrm{MHz}, \mathrm{CDCl}_{3}\right) \delta 7.42(\mathrm{~m}, 2 \mathrm{H})$, $7.28(\mathrm{~m}, 3 \mathrm{H}), 6.24(\mathrm{~m}, 1 \mathrm{H}), 5.66(\mathrm{~d}, \mathrm{~J}=15.85,1 \mathrm{H}), 2.15(\mathrm{q}, 2 \mathrm{H}), 1.42-1.29(\mathrm{~m}, 8 \mathrm{H}), 0.89$ $(\mathrm{t}, 3 \mathrm{H})$.

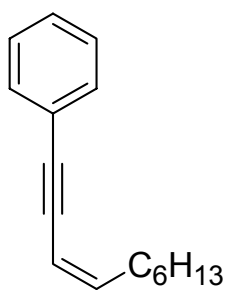

Dec-3-en-1-ynyl-benzene (Table 4, entry 3): The modified procedure was used to convert (Z)-1-lodo-oct-1-ene and phenyl acetylene to the title product. Purification by flash chromatography (hexane as the eluent) gave the analytically pure product as a colorless oil (420 mg, 98\% yield). ${ }^{1} \mathrm{H}$ NMR $\left(400 \mathrm{MHz}, \mathrm{CDCl}_{3}\right) \delta$ 7.44-7.41 (m, 2H), 7.29- 
$7.27(\mathrm{~m}, 3 \mathrm{H}), 5.99-5.92(\mathrm{~m}, 1 \mathrm{H}), 5.67-5.65(\mathrm{~d}, \mathrm{~J}=10.7,1 \mathrm{H}), 2.42-2.36(\mathrm{~m}, 2 \mathrm{H}), 1.46-1.43$ $(\mathrm{m}, 2 \mathrm{H}), 1.37-1.29(\mathrm{~m}, 6 \mathrm{H}), 0.89-0.86(\mathrm{~m}, 3 \mathrm{H}) .{ }^{13} \mathrm{C} \mathrm{NMR}\left(100 \mathrm{MHz}, \mathrm{CDCl}_{3}\right) \delta$ 144.36, 131.42, 128.30, 127.96, 123.82, 109.06, 93.46, 86.57, 31.76, 30.45, 28.96, 28.91, 22.70, 14.16. Anal. Calcd. for $\mathrm{C}_{16} \mathrm{H}_{20}$ : C, 90.51; H, 9.49; Found C, 90.24; H, 9.47.

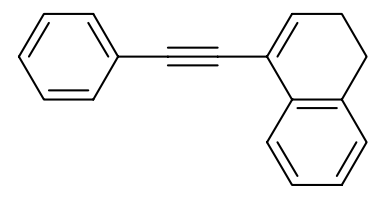

\section{1,2-dihydro-4-(2-phenylethynyl)naphthalene (Table 4, entry 4): The modified} procedure was used to convert phenylacetylene and 1,2-dihydro-4-iodonaphthalene to the title product in 24 hours. Purification by flash chromatography $\left(20 \% \mathrm{CH}_{2} \mathrm{Cl}_{2}\right.$ in hexanes) gave the analytically pure product as a light yellow oil (360 mg, $78 \%$ yield). ${ }^{1} \mathrm{H}$ NMR $\left(400 \mathrm{MHz}, \mathrm{CDCl}_{3}\right) \delta 7.68(\mathrm{~d}, \mathrm{~J}=7.58,1 \mathrm{H}), 7.52(\mathrm{~m}, 2 \mathrm{H}), 7.31(\mathrm{~m}, 3 \mathrm{H}), 7.25(\mathrm{~m}$, $1 \mathrm{H}), 7.18(\mathrm{t}, 1 \mathrm{H}), 7.13(\mathrm{~d}, J=7.33,1 \mathrm{H}), 6.54(\mathrm{t}, 1 \mathrm{H}), 2.80(\mathrm{t}, 2 \mathrm{H}), 2.42,(\mathrm{~m}, 2 \mathrm{H}) \cdot{ }^{13} \mathrm{C}$ NMR $\left(100 \mathrm{MHz}, \mathrm{CDCl}_{3}\right) \delta 135.49,135.05,132.64,131.54,128.29,128.07,127.66,127.39$, $126.62,125.05,123.43,121.72,90.28,87.29,27.14,23.69$. Anal. Calc'd. for $\mathrm{C}_{18} \mathrm{H}_{14}: \mathrm{C}$, 93.87; $\mathrm{H}, 6.13$; Found $\mathrm{C}, 93.79 ; \mathrm{H}, 6.36$.

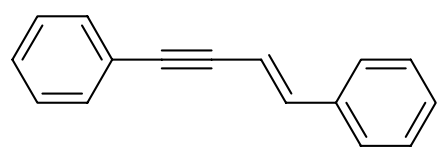

(E)-1,4-diphenylbutenyne (Table 4, entry 5): The modified procedure was used to convert phenyl acetylene and $\beta$-iodostyrene to the title product. Purification by flash chromatography (20\% $\mathrm{CH}_{2} \mathrm{Cl}_{2}$ in hexanes) gave the analytically pure product as a light yellow solid (399 mg, 98\% yield). ${ }^{1} \mathrm{H}$ NMR $\left(400 \mathrm{MHz}, \mathrm{CDCl}_{3}\right) \delta 7.47(\mathrm{~m}, 2 \mathrm{H}), 7.43(\mathrm{~d}, \mathrm{~J}=$ 7.22, 2H), 7.4-7.27 (m, 6H), $7.03(\mathrm{~d}, \mathrm{~J}=16.24,1 \mathrm{H}), 6.37$ (d, J=16.24). ${ }^{13} \mathrm{C}$ NMR (100 $\left.\mathrm{MHz}, \mathrm{CDCl}_{3}\right) \delta 141.24,136.31,131.50,128.71,128.60,128.32,128.16,126.29,123.42$, 108.13, 91.75, 88.91. Anal. Calcd. for $\mathrm{C}_{16} \mathrm{H}_{12}: \mathrm{C}, 94.08 ; \mathrm{H}, 5.92$; Found $\mathrm{C}, 93.96 ; \mathrm{H}$, 6.10. m.p. : $97-98^{\circ} \mathrm{C}\left(\right.$ lit: $\left..^{7} 96-97^{\circ} \mathrm{C}\right)$

References: 
(1) Piers, E.; Wong, T.; Coish, P. D.; Rogers, C. Can. J. Chem. 1994, 72, 1816-1819.

(2) Han, C.; Shen, R. C.; Su, S.; Porco, J. A. Org Lett. 2004, 6, 27-30.

(3) Lee, K.; Wiemer, D. F. Tetrahedron Lett. 1993, 34, 2433-2436.

(4) Brown, H. C.; Subrahmanyam, C.; Hamaoka, T.; Ravindran, N.; Bowman, D. H.; Misumi, S.; Unni, M. K.; Somayaji, V.; Bhat, N. G. J. Org. Chem. 1989, 54, 6068-6075.

(5) Bates, C. G.; Saejueng, P.; Murphy, J. M.; Venkataraman, D. Org. Lett. 2002, 4, 4727-4729.

(6) Kubas, G. J. Inorg. Synth. 1979, 19, 90-92.

(7) Dabdoub, M. J.; Dabdoub, V. B.; Comasseto, J. V. Tetrahedron Lett. 1992, 33, 2261-2264. 